\title{
DYNAMIC SIGNATURE ASSIGNMENT FOR REVERSE-LINK CDMA SYSTEMS
}

\author{
Jiunn-Tsair Chen ${ }^{1}$, Constantinos B. Papadias ${ }^{2}$ and Gerard J. Foschini ${ }^{2}$ \\ Dept. of Electronic Engineering ${ }^{1}$ \\ National Taiwan University of Sci. and Tech. \\ Taipei 106, Taiwan, ROC \\ Crawford Hill Laboratory ${ }^{2}$ \\ Bell Laboratories, Lucent Technologies \\ Holmdel, NJ 07733
}

\begin{abstract}
To guarantee low correlations among user signatures in the reverse link at the base station, in this paper, we proposed an adaptive algorithm to jointly update the user signatures. Making use of the wireless channel information, we iteratively reassign the spreading signature of the users with the minimum SINR (signal to interference-plus-noise ratio). As shown by simulation results, the proposed algorithm is likely to provide signatures which are orthogonal among users if the number of users is small, then multi-user detection is no longer required. When the number of users is large, the proposed algorithm still tries to maintain equal distances among user signatures, and thus improves the condition of the channel matrix and brings about an increase of capacity by up to 3-4 times, as compared to the same receivers with random codes.
\end{abstract}

\section{INTRODUCTION}

In DS-SS CDMA systems, each user is assigned a different spreading code over a wide frequency band. Each spreading code is then used to despread signals for the corresponding user. Therefore, the correlations among spreading codes or among the user signatures directly affect the interference between users. Orthogonal signatures eliminate all the interference among users. However, having a set of orthogonal signatures on the transmission side does not ensure that the user signatures seen on the receiver side are orthogonal, as each user experiences different inter-symbol interference (ISI), different propagation delays, and also different multipath propagation. Long pseudo-random codes are thus used in the reverse link of current CDMA systems [1]. Since the period of the pseudo code is usually much longer than the number of chips in a data symbol period, we can assume random codes are actually used in the reverse link for different users. To mitigate the high interference caused by the random codes, outer layer codings, such as convolution coding, are usually required.

Rake receivers are adopted in IS-95 [1], and widely deployed in the current DS-SS CDMA systems. This receiver structure is often called single-user detector so as to be distinguished from many other recently-proposed multi-user receiver structures which jointly demodulate the signals of multiple users. Among the multi-user detectors, the complexity of the optimal maximum likelihood (ML) receivers [2] grows exponentially with the number of users, and is considered too complicated to implement in practice. Suboptimum receiver structures include the linear approaches, such as decorrelator, MMSE (minimum mean square error) detectors $[3,4]$; and the non-linear apprciaches, such as decision-feedback detectors [5], multi-stage successive or parallel interference cancellation structures [6].

In the current CDMA system, the number of users loaded is only a small fraction of the number of chips $N$ within each symbol. This is because when the random codes are used to construct the user signatures, the interference among users is often enormous. A smaller number of users in the system can reduce the occurrence of high correlations among the user signatures. When the number of users is close to $N$, high interference is obvious in the single-user detector. Even the multi-user detectors cannot effectively surpress the interference because the channel matrix tends to be illconditioned. One way to get around this problem is to increase the dimension of the user signature by introducing antenna arrays into the system. If $P$ antennas are used, the dimension of the user signature increases from $N$ to $P N$. Therefore, the capacity is expected to increase linearly with the number of antenna elements [7]. Another way to get around this problem is to enforce the orthogonality or at least to reduce the cross-correlations among user signa.tures seen at the receiver.

In this paper, we propose an adaptive algorithm for the reverse link of a CDMA system to jointly optimize the user signatures seen at the receiver. An antenna array is used at the base station, but is not necessarily required in the proposed algorithm. With channel information availajle at the base station, we iteratively update the user signa sures. These user signature are then fed back to the corresponding users through the forward link. The organization of this paper is as follows: We first describe the system model in Section 2. In Section 3, we propose a dynamic signature assignment algorithm. Then, simulation results are presented in Section 4.

\section{SYSTEM MODEL}

The radio channel in a wireless communication system is often described by a multi-path propagation model. In large cells with high base station antenna platforms, the propagation environment is aptly modeled by a few dor.inant specular paths. In such a case, the baseband signal received 
at the antenna array can be expressed as follows, [8]

$$
\mathbf{x}(t)=\sum_{k=1}^{K} \sum_{l=1}^{L} \mathbf{a}\left(\theta_{k, l}\right) \beta_{k, l}(t) \tilde{s}_{k}\left(t-\tau_{k, l}\right)+\mathbf{n}(t)
$$

where $\mathbf{x}(t)$ is the received baseband signal; $\mathbf{a}\left(\theta_{k, l}\right)$ is the steering vector of user $k$ to a signal arriving from direction $\theta_{k, l} ; \beta_{k, l}$ is the complex time-varying amplitude of the $l^{\text {th }}$ path of the $k^{\text {th }}$ user, with the path amplitude being a complex Gaussian random process including both the propagation loss and the signal fading caused by the Doppler spread; $\tilde{s}_{k}$ is the transmitted complex baseband signal from the $k^{t h}$ user; $\tau_{k, l}$ is the propagation delay of the $l^{\text {th }}$ path of the $k^{t h}$ user; and $L$ is the maximum number of paths present in the system for each user. Note that we define $K$ as the number of users in the system visible to the base station employing the proposed algorithm. We assume the first $K_{0}$ users to be the in-cell users. The other $K-K_{0}$ users are out-of-cell users which generate enough interference to other in-cell users and affect our user signature optimization. However, the signals form the out-of-cell users are not demodulated at the base station.

In the DS-SS CDMA system with the length of the spreading code $N$ equal to the number of chips in a symbol period, the transmitted signal $\tilde{s}$ can be represented as the convolution of the data bits, the spreading codes and the pulse-shaping function. Assuming band-limited signals in the system, if we sample each continuous signal higher than twice the Nyquist rate, then the convolution, correlation, and integration operations can be replaced by multiplication of matrices with appropriate arrangements of matrix elements from the sampled data. In this system, we assume $P$ antennas, and oversample the signals with a factor of $\rho$. In addition, there are $N$ chips in each data symbol, and $M$ data symbols are considered. We also assume that the maximum path delay plus the length of the pulse-shaping function is no greater than $Q T_{\mathrm{c}}$ and that $Q<N$, where $T_{\mathrm{c}}$ is the chip period. If $Q$ is much smaller than $N$, then the system is quasi-synchronous. Therefore, after sampling, we can express the received signal $\mathbf{X}$ as

$$
\mathbf{X}=\sum_{k=1}^{K} \mathbf{A}_{k} \mathbf{B}_{k} \mathbf{G}_{k} \mathbf{S}_{k} \mathbf{D}_{k}+\mathbf{N}
$$

where $\mathrm{X}: P \times M N \rho$ and $\mathrm{N}: P \times M N \rho$ are, respectively, the received signal and the additive Gaussian noise; $\mathbf{A}_{k}: P \times L$ is given by $\mathbf{A}_{k}=\left[\mathbf{a}\left(\theta_{k, 1}\right) \mathbf{a}\left(\theta_{k, 2}\right) \ldots \mathbf{a}\left(\theta_{k, L}\right)\right] ; \mathbf{B}_{k}: L \times L$ is given by $\mathbf{B}_{k}=\operatorname{diag}\left\{\boldsymbol{\beta}_{k}\right\}$ with $\boldsymbol{\beta}_{k}=\left[\boldsymbol{\beta}_{k, 1}, \boldsymbol{\beta}_{k, 2}, \ldots, \boldsymbol{\beta}_{k, L}\right]^{T}$; and the $i, j^{\text {th }}$ element of $\mathbf{G}_{k}: L \times Q \rho$ can be written as $g\left(t_{0}+\frac{j-1}{\rho} T_{c}-\tau_{k, i}\right)$ with $g($.$) being the normalized pulse-$ shaping function. The code matrix $\mathbf{S}_{k}: Q \rho \times(N+Q-1) \rho$ is a Toeplitz matrix with $\left[s_{k, 1}, \mathbf{0}_{1 \times(Q \rho-1)}\right]^{T}$ as its first column, and $\left[s_{k, 1}, \mathbf{0}_{1 \times(\rho-1)}, s_{k, 2}, \mathbf{0}_{1 \times(\rho-1)}, \ldots, s_{k, N}, \mathbf{0}_{1 \times(Q \rho-1)}\right.$ as its first row, where $s_{k, 1}, s_{k, 2}, \ldots, s_{k, N}$ is the normalized spreading code of the $k^{t h}$ user. The data matrix $\mathbf{D}_{k}:(N+Q-1) \rho \times M N \rho$ is also a Toeplitz matrix with $\left[d_{k, m}, \mathbf{0}_{1 \times(N \rho-1)}, d_{k, m-1}, \mathbf{0}_{1 \times((Q-1) \rho-1)}\right]^{T}$ as its first column, and $\left[d_{k, m}, \mathbf{0}_{1 \times(N \rho-1)}, d_{k, m+1}, \mathbf{0}_{1 \times(N \rho-1)}, \ldots, d_{k, m+M-1}, \mathbf{0}_{1 \times(N \rho-1)}\right]$ as its first row, where $\left\{d_{k, m}\right\}$ are the BPSK (binary phase shift keying) data bits of the $k^{\text {th }}$ user. In addition, we define the temporal signature matrix $\tilde{\mathbf{S}}_{k}: L \times(N+Q-1) \rho$ as $\tilde{\mathbf{S}}_{k}=\mathbf{G}_{k} \mathbf{S}_{k}$. The $l^{t h}$ row of $\tilde{\mathbf{S}}_{k}$ denotes the temporal signature of the $l^{\text {th }}$ path of the $k^{\text {th }}$ user seen at the receiver.

\section{THE ALGORITHM}

In this section, we describe a novel algorithm to adaptively update the user signatures in the reverse link of the CDMA system in order to reduce the mutual interference among users. This thus improves the performance and increases the system capacity, because the CDMA systems are usually interference-limited. The received signals are first used for joint channel estimation. Based on the channel estimates, we adaptively optimize the spreading codes and the transmission delays. In demodulating the received baseband signals in the reverse link, the information of the channel estimates and the optimized user signatures are used in the space-time matched filter and the linear receiver (or simply slicers for the single-user detector). At the same time, the optimized spreading codes and the transmission delays are fed back to the mobile users through the forward link to update their user signatures for the next round of transmission. The space-time matched filer, the linear receivers, and the adaptive signature optimization scheme are, respectively, discussed in more detail in the following sub-sections.

\subsection{Space-Time Matched Filters}

The space-time matched filter is usually used to obtain the sufficient statistics for demodulating digitally modulated signals. The output of the matched filter can be written as

$$
\overline{\mathbf{X}}_{k, m}=\operatorname{Re}\left\{\operatorname{tr}\left\{\mathbf{B}_{k}^{*} \mathbf{A}_{k}^{*} \mathbf{X}^{m} \tilde{\mathbf{S}}_{k}^{*}\right\}\right\} .
$$

where "tr" denotes the trace of a matrix, and $\mathbf{X}^{m}: P \times$ $(N+Q-1) \rho$ is copied from the $((m-1) N \rho+1)^{t h}$ to $(m N \rho+Q \rho)^{t h}$ columns of $\mathbf{X}$. The matrix multiplication of $\mathbf{A}_{k}^{*}$ and $\mathbf{X}^{m}$ denotes the path-wise beam-forming. Let $\tilde{\mathbf{X}}_{k, m}=\mathbf{A}_{k}^{*} \mathbf{X}^{m}$. The trace of the matrix multiplication $\operatorname{tr}\left\{\mathbf{B}_{k}^{*} \tilde{\mathbf{X}}_{k, m} \mathbf{S}_{k}^{*}\right\}$ denotes the matched filter for the temporal signature and the maximum-ratio path combining of the $k^{t h}$ user. To expand $\overline{\mathbf{X}}_{k, m}$, (3) can be rewritten as

$$
\begin{aligned}
\overline{\mathbf{X}}_{k, m} & =\sum_{i=1}^{K} \operatorname{Re}\left\{\operatorname{tr}\left\{\mathbf{B}_{k}^{*} \mathbf{A}_{k}^{*} \mathbf{A}_{i} \mathbf{B}_{i} \tilde{\mathbf{S}}_{i} \mathbf{D}_{i}^{m} \tilde{\mathbf{S}}_{k}^{*}\right\}\right\} \\
& +\operatorname{Re}\left\{\operatorname{tr}\left\{\mathbf{B}_{k}^{*} \mathbf{A}_{k}^{*} \mathbf{N}^{m} \tilde{\mathbf{S}}_{k}^{*}\right\}\right\},
\end{aligned}
$$

where as $\mathbf{N}^{m}$ and $\mathbf{D}_{i}^{m}$ are, respectively, the submatrices of $\mathbf{N}$ and $\mathbf{D}_{i}$, so $\mathbf{X}^{m}$ is constructed from $\mathbf{X}$. Note that $\mathbf{D}_{i}^{m}$ is a Toeplitz square matrix with $\left[d_{i, m}, \mathbf{0}_{1 \times(N \rho-1)}, d_{i, m-1}, \mathbf{0}_{1 \times((Q-1) \rho-1)}\right]^{T}$ as its first column, and $\left[d_{i, m}, \mathbf{0}_{1 \times(N \rho-1)}, d_{i, m+1}, \mathbf{0}_{1 \times((Q-1) \rho-1)}\right]$ as its first row. Therefore, the first term on the right hand side of (4) can be written as

$$
\begin{aligned}
\overline{\mathbf{X}}_{k, m} & =\sum_{i=1}^{K} \operatorname{Re}\left\{d_{k, m} \operatorname{tr}\left\{\mathbf{B}_{k}^{*} \mathbf{A}_{k}^{*} \mathbf{A}_{i} \mathbf{B}_{i} \tilde{\mathbf{S}}_{i} \tilde{\mathbf{S}}_{k}^{*}\right\}\right. \\
& +\bar{d}_{k, m-1} \operatorname{tr}\left\{\mathbf{B}_{k}^{*} \mathbf{A}_{k}^{*} \mathbf{A}_{i} \mathbf{B}_{i} \tilde{\mathbf{S}}_{i}(+1) \tilde{\mathbf{S}}_{k}^{*}(-1)\right\} \\
& \left.+d_{k, m+1} \operatorname{tr}\left\{\mathbf{B}_{k}^{*} \mathbf{A}_{k}^{*} \mathbf{A}_{i} \mathbf{B}_{i} \tilde{\mathbf{S}}_{i}(-1) \tilde{\mathbf{S}}_{k}^{*}(+1)\right\}\right\}
\end{aligned}
$$




$$
\begin{aligned}
& =\sum_{i=1}^{K} \operatorname{Re}\left\{d_{k, m} \boldsymbol{\beta}_{k}^{*}\left(\left(\mathbf{A}_{k}^{*} \mathbf{A}_{i}\right) \odot\left(\tilde{\mathbf{S}}_{i} \tilde{\mathbf{S}}_{k}^{*}\right)^{T}\right) \boldsymbol{\beta}_{i}\right. \\
& +d_{k, m-1} \boldsymbol{\beta}_{k}^{*}\left(\left(\mathbf{A}_{k}^{*} \mathbf{A}_{i}\right) \odot\left(\tilde{\mathbf{S}}_{i}(+1) \tilde{\mathbf{S}}_{k}^{*}(-1)\right)^{T}\right) \boldsymbol{\beta}_{i} \\
& \left.+d_{k, m+1} \boldsymbol{\beta}_{k}^{*}\left(\left(\mathbf{A}_{k}^{*} \mathbf{A}_{i}\right) \odot\left(\tilde{\mathbf{S}}_{i}(-1) \tilde{\mathbf{S}}_{k}^{*}(+1)\right)^{T}\right) \boldsymbol{\beta}_{i}\right\}(5)
\end{aligned}
$$

where $\odot$ denotes the Hadamard product or the entry-wise product [9], $\tilde{\mathbf{S}}_{k}(+1)$ and $\tilde{\mathbf{S}}_{k}(-1)$ are both sub-matrices of $\tilde{\mathbf{S}}_{k}$. The rightmost and the leftmost $(Q-1) \rho$ columns of $\tilde{\mathbf{S}}_{k}$ are respectively denoted by $\tilde{\mathbf{S}}_{k}(+1)$ and $\tilde{\mathbf{S}}_{k}(-1)$.

We can stack the matrices and write the received signal at the output of the matched filter as

$$
\overline{\mathbf{X}}=\mathcal{H} \mathbf{d}+\overline{\mathbf{N}},
$$

where $\overline{\mathbf{X}}=\left[\overline{\mathbf{X}}_{1,1}, \overline{\mathbf{X}}_{2,1}, \ldots, \overline{\mathbf{X}}_{K, 1}, \overline{\mathbf{X}}_{1,2}, \ldots, \overline{\mathbf{X}}_{K, M}\right]^{T}, \mathbf{d}=$ $\left[d_{1,1}, d_{2,1}, \ldots, d_{K, 1}, d_{1,2}, \ldots, d_{K, M}\right]^{T}, \quad$ and $\overline{\mathbf{N}}=\left[\overline{\mathbf{N}}_{1,1}, \overline{\mathbf{N}}_{2,1}, \ldots, \overline{\mathbf{N}}_{K, 1}, \overline{\mathbf{N}}_{1,2}, \ldots, \overline{\mathbf{N}}_{K, M}\right]^{T}$ with $\overline{\mathbf{N}}_{k, m}=$ $\operatorname{Re}\left\{\operatorname{tr}\left\{\mathbf{B}_{k}^{*} \mathbf{A}_{k}^{*} \mathbf{N}^{m} \overline{\mathbf{S}}_{k}^{*}\right\}\right\}$. If we define $\mathbf{H}(0): K \times K, \mathbf{H}(+1):$ $K \times K$, and $\mathbf{H}(-1): K \times K$ as

$$
\begin{aligned}
\mathbf{H}(0) & =\mathbf{B}^{*}\left(\left(\mathbf{A}^{*} \mathbf{A}\right) \odot\left(\tilde{\mathbf{S}} \tilde{\mathbf{S}}^{*}\right)^{T}\right) \mathbf{B} \\
\mathbf{H}(+1) & =\mathbf{B}^{*}\left(\left(\mathbf{A}^{*} \mathbf{A}\right) \odot\left(\tilde{\mathbf{S}}(+1) \tilde{\mathbf{S}}^{*}(-1)\right)^{T}\right) \mathbf{B}, \\
\mathbf{H}(-1) & =\mathbf{B}^{*}\left(\left(\mathbf{A}^{*} \mathbf{A}\right) \odot\left(\tilde{\mathbf{S}}(-1) \tilde{\mathbf{S}}^{*}(+1)\right)^{T}\right) \mathbf{B}
\end{aligned}
$$

$\underset{\tilde{\mathbf{S}}}{\text { with }} \mathbf{A}=\left[\mathbf{A}_{1}, \mathbf{A}_{2}, \ldots, \mathbf{A}_{K}\right], \quad \tilde{\mathbf{S}}=\left[\tilde{\mathbf{S}}_{1}^{T}, \tilde{\mathbf{S}}_{2}^{T}, \ldots, \tilde{\mathbf{S}}_{K}^{T}\right]^{T}$, $\tilde{\mathbf{S}}(+1)=\left[\hat{\mathbf{S}}_{1}^{T}(+1), \ddot{\mathbf{S}}_{2}^{T}(+1), \ldots, \tilde{\mathbf{S}}_{K}^{T}(+1)\right]^{T}, \tilde{\mathbf{S}}(-1)=$ $\left[\tilde{\mathbf{S}}_{1}^{T}(-1), \tilde{\mathbf{S}}_{2}^{T}(-1), \ldots, \mathbf{S}_{K}^{T}(-1)\right]^{T}$, and define the fading amplitude matrix $\mathbf{B}: K L \times K$ as $\mathbf{B}=\operatorname{diag}\left(\boldsymbol{\beta}_{1}, \boldsymbol{\beta}_{2}, \ldots, \boldsymbol{\beta}_{K}\right)$, then $\mathcal{H}$ can be expressed as

$$
\mathcal{H}=\operatorname{Re}\left\{\left[\begin{array}{ccccc}
\mathbf{H}(0) & \mathbf{H}(-1) & & & \mathbf{0} \\
\mathbf{H}(+1) & \mathbf{H}(0) & \mathbf{H}(-1) & & \\
& \mathbf{H}(+1) & \mathbf{H}(0) & \mathbf{H}(-1) & \\
& \vdots & \vdots & \vdots & \\
\mathbf{0} & & & \mathbf{H}(+1) & \mathbf{H}(0)
\end{array}\right]\right\}
$$

\subsection{Signal Detectors}

Recall from Section 2 that only $K_{0}$ in-cell users need to be demodulated at the base station. Therefore, only the matched filter outputs related to these $K_{0}$ users are processed in the signal detector. In this subsection, we describe three signal detectors, which we use in this paper to test our proposed algorithm.

1. Single-user detector: $K_{0}$ slicers are used at each output of the matched filter. Decisions are instantaneous with no delay. The data estimate is given by

$$
\hat{d}_{k, m}=\operatorname{sgn}\left(\overline{\mathbf{X}}_{k, m}\right)
$$

where $\overline{\mathbf{X}}_{k, m}$ is the output of the matched filter in (3).

2. Decorrelator: A zero-forcing solution is applied to solve (6). The data estimate can then be written as

$$
\hat{\mathbf{d}}=\mathcal{H}^{+} \overrightarrow{\mathbf{x}} \text {. }
$$

For the decorrelator and the MMSE detector described next, the maximum possible delay could be up to $M$ symbols because of the block processing of the asynchronous signals.
3. MMSE detector: Taking into account the effect of the color noise, MMSE solution usually performs better than the zero-forcing solution of the decorrelator. The coloring matrix of the noise, $\overline{\mathbf{N}}$ in (6), at the output of the matched filter is given by

$$
\mathrm{E}\left(\overline{\mathbf{N}} \overline{\mathbf{N}}^{*}\right)=\frac{\sigma_{n}^{2}}{2} \mathcal{H}
$$

The data estimate for the MMSE detector can then be expressed as

$$
\hat{\mathbf{d}}=\mathcal{H}^{*}\left(\mathcal{H} \mathcal{H}^{*}+\frac{\sigma_{n}^{2}}{2 \sigma_{b}^{2}} \mathcal{H}\right)^{-1} \overline{\mathbf{X}}
$$

where $\sigma_{n}^{2}$ denotes the noise variance chosen to match the desired $\frac{E_{b}}{N_{0}}$ with $E_{b}$ being the bit energy and $N_{0}$ being the average noise variance, and $\sigma_{b}^{2}=1$ for the BPSK modulation scheme.

\subsection{Signature Searching Strategies}

With the information of the channel estimate available at the base station, we try to optimize the user signature so as to minimize the mutual interference among users. One way to suppress the mutual interference is to maximize the minimum SINR $\xi_{\text {min }}$ among in-cell users,

$$
\xi_{\min }=\min _{k=1,2, \ldots, K_{0}} \xi_{k} .
$$

The SINR of the $k^{t h}$ user $\xi_{k}$ can be written as the power ratio of the signal and the interference-plus-noise:

$$
\xi_{k}=\frac{\xi_{S, k}}{\xi_{I, k}+\xi_{N, k}}
$$

The signal power $\boldsymbol{\xi}_{S}: K \times 1$ can be written as

$$
\boldsymbol{\xi}_{S}=\operatorname{diag}(\mathbf{H}(0)) \odot \operatorname{conj}(\operatorname{diag}(\mathbf{H}(0)),
$$

where $\mathbf{H}(0)$ is given in (7), "conj" is the complex conjugate operation, and the output of $\operatorname{diag}($.$) is a column vector$ consisting of the diagonal elements of the argument matrix. The interference power $\boldsymbol{\xi}_{I}: K \times 1$ can be written as

$$
\boldsymbol{\xi}_{I}=\operatorname{diag}\left(\mathbf{H H}^{*}\right)-\boldsymbol{\xi}_{S},
$$

where $\mathbf{H}=[\mathbf{H}(-1), \mathbf{H}(0), \mathbf{H}(+1)]$, and $\mathbf{H}(-1), \mathbf{H}(0)$ and $\mathbf{H}(+1)$ are given in (7) - (9). The noise power $\boldsymbol{\xi}_{N}: K \times 1$ can be written as

$$
\boldsymbol{\xi}_{N}=\frac{\sigma_{n}^{2}}{2} \operatorname{diag}(\mathbf{H}(0))
$$

Note that in using the minimum SINR as the criterion for signature optimization, two factors are taken into consideration: 1)the received power of each user at the base station, and 2) the orthogonality among user signatures.

Since some out-of-cell users may generate as strong interference to the other users as those in-cell users, we should not restrict our handling of signature optimization only to users within the cell. However, to update signatures of outof-cell users requires coordination among base stations. Updating a specific out-of-cell user signature may reduce the 
interference at the in-cell base station, but high interferences may be generated at the same time at the base station of a neighboring cell. In this paper, we assume $K=K_{0}$, i.e., the only visible users are those in-cell users. For those systems with high frequency re-use factor, $K=K_{0}$ is a reasonable assumption. The procedure and the performance of the proposed algorithm when applied to the general cases where $K>K_{0}$ will be discussed in another paper.

Before we move on to the discussion of the optimization of user signatures, the following observations should be noted:

1. Random search: We need to explore the less dense regions of the signal space to maximize the distances among the user signatures. Namely, we need to adopt some of those un-used signatures by random search in order to span the whole signal space.

2. Gradient search: After obtaining a signature in a less dense area in the signal space by random search, we can further optimize this signature by gradient-searching the neighborhood of this area. It is unlikely to reach the best signature only by random search, but the best signature available is likely to be around the less dense areas.

3. Target user isolation: Instead of updating all the signatures for all the users every time, we choose to isolate a target user each time and optimize its signature before moving on to optimize other user signatures. Isolating a target user each time not only speeds up convergence but also reduces the algorithm complexity.

4. Sequential packing: The signature assignment is more likely to converge to the optimum solution when the number of users is small. Furthermore, the optimal signature assignment of a $K$-user system is likely to be similar to that of a $(K-1)$-user system. Therefore, we suggest that users be added into the system one by one. A new user is introduced into the system every time when the signature optimization procedure is converged in the previous system.

Based on the observation above, we developed a signature optimization procedure. We start from the system with only one user. We add a new user into the system and start a new cycle of signature optimization procedure only when the previous cycle of signature optimization is converged. In each cycle, we first pick a target user, optimize the signature of this user by both random search and gradient search, then test if the search is converged. We define convergence of signature search as the condition where there is no space for further improvement no matter which target user we pick. If it is not converged, we pick another target user. If it is converged, we add a new user to the system until the total number of users reaches $K$.

\section{SIMULATION RESULTS}

Simulations were conducted to compare the performance of the proposed algorithm with dynamic signature assignment and the conventional algorithm with random spreading codes and transmission delays. Three receivers, singleuser detector, decorrelator, and MMSE detector, are tested and their corresponding BERs (bit error rate) are collected.
The simulations test the DS-SS CDMA signals used in the reverse link IS-95 system [1]. We approximate the long pseudo-random codes crossing many data symbols by a short random code extending only one data symbol. However, in evaluating BER, we averaged the BER over the possible random codes. Each random codes is eight chips long. Square root raised-cosine pulse-shaping function with roll-off factor 0.35 is used in the front end chip modulation. Users are uniformly distributed within a 120-degree sector. We use a uniform linear array with 4 isotropic elements spaced $\frac{1}{\sqrt{3}}$ wavelength apart. Each path is assumed to be a specular path as describe in Section 2. The maximum path delays among all the paths are assumed to be within a symbol period in an asynchronous system. The fading amplitudes are independent between paths. The complex path fading amplitudes are described as zero mean complex circularly distributed Gaussian random variables that are mutually independent between real parts and imaginary parts. They are held constant over each signature updating $c y$ cle. In order to isolate the effect of the proposed algorithm from the power control problem, a perfect power control is assumed. The additive noise is modeled as a white conplex circularly distributed Gaussian random variable with zero mean. The real and imaginary parts of the noise each has variance $\frac{\sigma_{n}^{2}}{2}$, where $\sigma_{n}^{2}$ is varied to give the desired $\frac{E_{b}}{N_{0}}$. We average the BER over 500 independent trials for each algorithm.

In the simulation results, we focus on the BERs of the worst-performing user in the system. We compare these BERs of various algorithms with 32 users in the system and with different $\frac{E_{b}}{N_{0}}$ in Fig. 1. We also compare the same BERs of the same algorithms with different numbers of users in the system and with $\frac{E_{b}}{N_{0}}=0 d B$ in Fig. 2. In Fig. 1, the proposed algorithm almost always performs better than the conventional algorithm without signature optimization regardless of the choice of the detector. Except for the MMSE detector, the conventional algorithm with other detectors performs very poorly. This is because we have 32 users in a system using only 4 antennas and eightchip-long spreading code. Too many users in the system results in severe mutual interference among users, and causes the channel matrix $\mathcal{H}$ in (10) to be highly ill-conditioned. The BER curve of the single-user detector is basically flat, which means the interferences dominate the system performance. At low $\frac{E_{b}}{N_{0}}$, even the single-user detector performs better than the decorrelator which is also due to the channel matrix being ill-conditioned and thus causes severe noise enhancement problem. At higher $\frac{E_{b}}{N_{0}}$, decorrelation among user signatures helps to improve the performance. Note that the proposed algorithm with the MMSE detector approaches the single-user lower bound. However, the error flooring of the proposed algorithm with single-user detector implies that the proposed algorithm cannot always converge to the orthogonal solution in the signature assignment. If the proposed algorithm can converge to the solution where all the user signatures are orthogonal to one another, then the performance of the proposed algorithm should be exactly the same as that of the single-user lower bound regardless of the choice of the detector.

In Fig. 2, the proposed algorithm again almost always 
performs better than the conventional algorithm without signature optimization regardless of the choice of the detector. Comparing the BER curves of the same detector of the proposed algorithm and of the conventional algorithm, we find a capacity improvement of 3 to 4 times. In the conventional algorithm, the decorrelator perform almost as well as that of the MMSE detector when the number of users is small. However, when the number of users increases and approaches the dimension of the signal space, the decorrelator starts to perform worse than the single-user detector because of the noise enhancement problem mentioned earlier. In the proposed algorithm, the improvement from the single-user detector to the multi-user detector indicates that some of the converged solutions are not orthogonal among user signatures when the number of users is large.

\section{CONCLUSION}

We have proposed a signature optimization algorithm, which adaptively reassign the spreading code and the transmission delay for each user in the system in order to reduce the mutual interference among users. The proposed algorithm guarantees convergence. With some strategies including 1) random search, 2) gradient search, 3) target user isolation, and 4) sequential packing, we make the signature searching more efficient and converge to a better solution. Furthermore, as shown by the simulation results, the proposed algorithm can provide capacity improvement of 3-4 times compared to the conventional algorithms without signature optimization regardless of the choice of the detectors.

\section{REFERENCES}

[1] TIA/EIA/IS95, "Mobile station -base station compatibility standard for dual-Mode wideband spread spectrum cellular system," Telecommunication Industry Association, July, 1993.

[2] Verdu, S., "Minimum probability of error for asynchronous Gaussian multiple-access channels," IEEE Transactions on Information Theory, vol.IT-32, no.1, p. 85-96, Jan. 1986.

[3] Miller,

S.Y.; Schwartz, S.C., "Integrated spatial-temporal detectors for asynchronous Gaussian multiple-access channels," IEEE Transactions on Communications, vol.43, no.24, pt.1, p. 396-411, Feb.-April 1995.

[4] Xie, Z.; Short, R.T.; Rushforth, C.K., "A family of suboptimum detectors for coherent multiuser communications," IEEE Journal on Selected Areas in Communications, vol.8, no.4, p. 683-90, May 1990.

[5] Duel-Hallen, A., "A family of multiuser decisionfeedback detectors for asynchronous code-division multiple-access channels," IEEE Transactions on Communications, vol.43, no.2-4, pt.1, p. 421-34, Feb.Apr. 1995.

[6] Varanasi, M.K.; Aazhang, B., "Multistage detection in asynchronous code-division multiple-access communications," IEEE Transactions on Communications, vol.38, no.4, p. 509-19, April 1990.

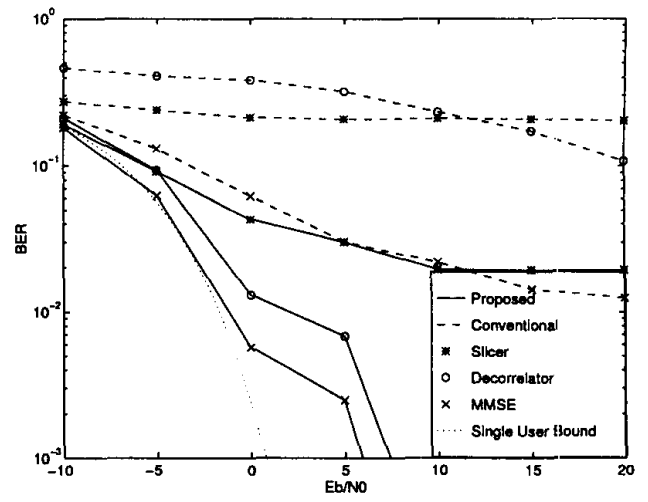

Figure 1. Performance comparison of various detectors for the proposed and conventional algorithms with 8-chip spreading codes, 4 antennas, and 32 users.

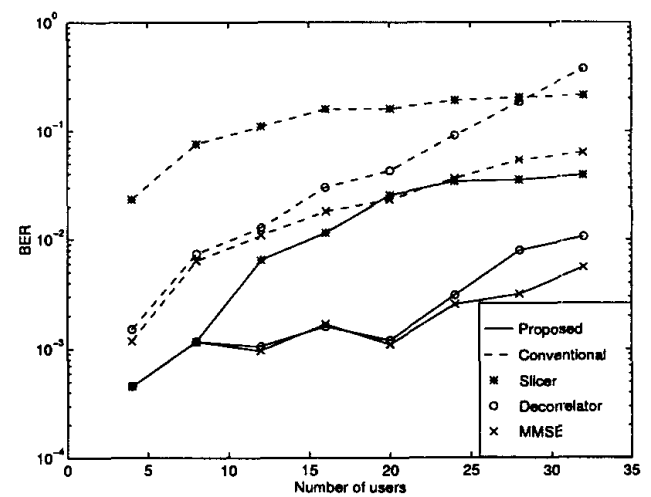

Figure 2. Performance comparison of various detectors for the proposed and conventional algorithms with 8-chip spreading codes, 4 antennas, and $\frac{E_{b}}{N_{0}}=0 d B$.

[7] Kandala, S.; Sousa, E.S.; Pasupathy, S., "Multi-user multi-sensor detectors for CDMA networks," 'EEE Transactions on Communications, vol.43, no.2-4, pt.2, p. 946-57, Feb.-March 1995.

[8] Raleigh, G.; Diggavi, Suhas N.; Naguib, Ayman F.; Paulraj, Arogyaswami, "Characterization of Fast Fading Vector Channels for Multi-Antenna Communication Systems," $28^{\text {th }}$ Asilomar Conference on Signals, Systems and Computers, 1994.

[9] Horn, Roger A.; Johnson, Charles R., "Topics in Matrix Analysis," Cambridge University Press, 1991. 